Journal of Applied Mathematics and Stochastic Analysis, 16:2 (2003), 127-139.

Printed in the USA (C)2003 by North Atlantic Science Publishing Company

\title{
TREE-INDEXED PROCESSES: A HIGH LEVEL CROSSING ANALYSIS ${ }^{1}$
}

\author{
MARK KELBERT \\ EBMS, University of Wales-Swansea, \\ Singleton Park, Swansea, SA2 8PP UK \\ and \\ YURI SUHOV \\ DPMMS, University of Cambridge, \\ Wilberforce Road, Cambridge, CB3 OWB, UK
}

(Received June 2002; Revised April 2003)

\begin{abstract}
Consider a branching diffusion process on $\mathbf{R}^{1}$ starting at the origin. Take a high level $u>0$ and count the number $R(u, n)$ of branches reaching $u$ by generation $n$. Let $F_{k, n}(u)$ be the probability $\mathbf{P}(R(u, n)<k), k=1,2, \ldots$ We study the $\operatorname{limit}_{n \rightarrow \infty} F_{k, n}(u)=$ $F_{k}(u)$. More precisely, a natural equation for the probabilities $F_{k}(u)$ is introduced and the structure of the set of solutions is analysed. We interpret $F_{k}(u)$ as a potential ruin probability in the situation of a multiple choice of a decision taken at vertices of a 'logical tree'. It is shown that, unlike the standard risk theory, the above equation has a manifold of solutions. Also an analogue of Lundberg's bound for branching diffusion is derived.

Keywords: Ruin Probability, Lundberg's Bound, Random Tree, Multiple Choice Potential Ruin, Dynamical System, Phase Portrait.
\end{abstract}

AMS (MOS) Subject Classification: 60J85, 60K10

\section{Introduction}

1. Recall the classical risk model (see, e.g., [2], [3]) : successive claims arrive to an insurance company according to a Poisson process $\mathbf{N}=(N(t), t \geq 0)$ of intensity $\lambda$. These claims form an i.i.d. sequence $X_{j}, j=1,2, \ldots$ (independent of the process $\mathbf{N}$ ) with the mean claim size $\mu_{1}$ and the second moment $m_{2}=E\left[X_{j}^{2}\right]$. On the other hand, the company has a deterministic income $c t$ during $[0, t]$, where $c$ is the gross premium rate. The probability of ruin $\psi(u)$, when the initial reserve equals $u$, is given by

$$
\psi(u)=\mathbf{P}(u+c t-S(t)<0 \quad \text { for some } t>0)=\mathbf{P}(L>u) .
$$

Here $S(t)=\sum_{j=1}^{N(t)} X_{j}$ is the claim process, $Z(t)=S(t)-c t$ and $L=\sup _{t>0} Z(t)$ is the maximal aggregate loss. Define the safety loading $\rho$ by the relation $c=(1+\rho) \lambda \mu_{1}$ (see $[6])$. Hereafter we assume that $\rho>0$.

\footnotetext{
${ }^{1}$ The work was partially done under the INTAS-00-265 Grant.
} 
The diffusion approximation for ruin probability may be defined as follows. Given $\gamma>0$, write:

$$
\psi(u)=\mathbf{P}\left(\sup _{t>0} Z(\gamma t)>u\right) .
$$

We want to scale the safety loading and the initial reserve so that $\rho=\frac{\bar{\rho}}{\sqrt{\gamma}}$ and $u=\mu_{1} \sqrt{\gamma}$ where $\gamma \rightarrow \infty$. One then can easily check the convergence $\psi(u) \rightarrow \psi_{D}$ where

$$
\psi_{D}=\mathbf{P}\left(\sup _{t>0}(W(t)+a t)>\frac{\mu_{1}}{\sqrt{\lambda m_{2}}}\right) .
$$

Here $W$ is a standard Wiener process, and the negative slope $a=-\bar{\rho} \mu_{1} \sqrt{\frac{\lambda}{m_{2}}}$.

A straightforward computation with the Wiener process (based on the reflection principle) gives that $\psi_{D}=\exp \left(\frac{2 a \mu_{1}}{\sqrt{\lambda m_{2}}}\right)$ (see, e.g., Formula 1.1.4 in Section 2, Ch.2, [5]). This leads directly to a 'diffusion version' of the famous Lundberg's bound (which now becomes an equality):

$$
\psi_{D}=\exp \left(-2 \bar{\rho} \mu_{1}^{2} / m_{2}\right)=\exp \left(-2 \rho \mu_{1} u / m_{2}\right)
$$

For the standard Lundberg's bound, see, e.g. [2], [3] or [6].

On the other hand, one can easily derive the diffusion Lundberg's formula (1.4) directly, using an explicit expression for the moment generating function (m.g.f.) of maximal aggregate loss $L$ in the original Poisson model. Namely,

$$
M_{L}(s)=\frac{\rho \mu_{1} s}{1+(1+\rho) \mu_{1} s-M_{X}(s)},
$$

and $M_{X}(s)$ is the m.g.f. of an individual claim size $X$ (see [6]). Using the scaling $L=\bar{L} \sqrt{\gamma}$ and $\rho=\frac{\bar{\rho}}{\sqrt{\gamma}}$ in (1.5) with $\gamma \rightarrow \infty$, one obtains in the limit the exponential distribution $P(\bar{L}>x)=\exp \left(-2 \bar{\rho} \mu_{1} x / m_{2}\right)$.

In this paper we consider a version of the classical risk theory model for the socalled tree-indexed processes where the one-dimensional time is replaced by a tree. Such an approach was developped in [8] where the concept of a potential ruin time was introduced and analysed. One of the results of the present paper is to establish, under an assumption about asymptotics of re-scaling probabilities, a diffusion analogue of Lundberg's bound in this situation. See Section 2.

2. Another topic discussed below is a modification of the potential ruin probability for branching diffusion processes. Fix an initial capital $u>0$ and consider a branching diffusion $\mathbf{Z}(t)=\left\{Z_{i}(t), i \leq I(t)\right\}, t \geq 0$, with constant drift $a$, diffusion coefficient $\sigma$ and fission rate $\varpi$ and two offspring replacing a single parent at each act of division. (Here $I(t)$ stands for the random size of population at time $t \geq 0$.) An interesting application arises when we interpret the values $u-Z_{i}(t)$ as a potential balance, at time $t$, if the insurance company decides to follow a policy represented by the path from the root of the binary tree to the 'leaf' corresponding to offspring $i \leq I(t)$. Such a policy is a result of subsequently taken decisions leading to one of two branches of the binary tree. See [8] for a detailed discussion.

Now, given $n=1,2, \ldots$, consider the number $R(u, n)$ of branches of the branching diffusion tree reaching $u$ at least once by the time of $n$-th fission. Set: $F_{n, k}(u)=$ $\mathbf{P}(R(u, n)<k), k=1,2, \ldots$ We study the limiting probabilities $F_{k}(u)=\lim _{n \rightarrow \infty} F_{n, k}(u)$. For example, in the above application, the difference $F_{k+1}(u)-F_{k}(u), \stackrel{n \rightarrow \infty}{k}>1$, gives 
the limiting probability, as $n \rightarrow \infty$, that there exist exactly $k$ 'dangerous' policies along which company's assets becomes threatened at least once before the time when the $n$-th decision has to be taken. The potential ruin probability (on the whole tree) studied in [8] equals $1-F_{1}(u)$. With regards to above applications, the values $F_{k}(u)$ give a detailed characterization of company's possible performance; we will call them multiple-choice potential ruin (m.c.p.r.) probabilities.

In fact, we introduce natural equations (see (3.9)) for probabilities $F_{k}(u)$ and analyse the structure of the set of solutions. The first (unsurprising) result here is that if $F_{1}(u) \equiv 0, u>0$, then $\forall k>1, F_{k}(u) \equiv 0, u>0$. A naive interpretation of this result is that if there exists a single branch of the tree reaching level $u$ then there exists at least $k$ such branches, for every $k$. However, an attempt to make such a statement precise by considering an infinite tree is misleading: if level $u$ is reached at some vertix then any branch of the infinite tree passing this vertex should be counted as a 'ruinous strategy'. The second (rather surprising) result is that the solutions to the above equations are nonunique. Analysing the non-uniqueness that arises here leads to interesting conclusions: there exist a family of m.c.p.r. probabilities whose values depend on conditions of the economic environment that may occur in a distant future.

On the other hand, all solutions to our equations have a number of common features, one of which is a particular logarithmic asymptotics as $u \rightarrow \infty$. The corresponding statements and arguments behind the proofs are given in Sections 3 and 4.

We want to mention that we consider here only one of the aspects of the (big and serious) problem of how a random tree (in particular, a trajectory of a branching diffusion) reaches a given level. Our results imply that the times when branches of the tree cross the level accumulate towards the 'terminal' time $n$, as $n \rightarrow \infty$. It is important to stress that although within our approach, there exist limits of probabilities under consideration as $n \rightarrow \infty$, we do not work with infinite trees. There are other versions of the same problem, stated in terms of an infinite tree, but they require different analytic techniques.

\section{Lundberg's Bound on a Tree}

We define the potential ruin time as the shortest length of a policy that ruins a company with the initial capital $u$ (cf. [8]):

$$
\theta(u)=\min \left[l \geq 1: \exists \quad \text { a path } \mathbf{L} \text { with } \quad \mathbf{L} \in \mathcal{L}_{l}, Z_{\mathbf{L}}>u\right]
$$

and the potential ruin probability as

$$
\psi(u)=\mathbf{P}(\theta(u)<\infty) .
$$

Here and below, $\mathcal{L}_{n}$ is a set of $2^{n+1}$ paths of length at most $n$ on a discrete tree of branching degree two and $\mathcal{L}$ is the set of all finite paths starting at the root of the infinite binary tree.

As was mentioned, a path $\mathbf{L} \in \mathcal{L}_{n}$ could be identified with a binary sequence $\left(j_{1}, \ldots, j_{n^{\prime}}\right), n^{\prime} \leq n$, of trading policies $j_{i}(=0,1)$ assigned to subsequent edges of the tree. Furthermore, $Z_{\mathbf{L}}$ represents the sum $\sum_{i=1}^{n^{\prime}} Z_{j_{i}}$ that gives the overall balance after $n^{\prime}$ trading periods. The random variables $Z_{j_{i}}$ are i.i.d. They are associated with edges and represent the local gain or loss after applying the corresponding trading policy $j_{i}$ during trading period $i$. 
Sufficient conditions for the bound $\psi(u)<1$ are presented in Section 2 of [8]. Here we give only a simple condition that guarantees the decrease of this probability in the discrete case using some basic facts and notations from the theory of branching random walks (see [4]). Denote by $G_{n}$ the $n$-th generation of initial particle and consider the function

$$
m(\kappa)=\mathbf{E}\left(\sum_{x \in G_{1}} \exp \left(\kappa Z_{x}\right)\right)
$$

where $Z_{x}, x \in G_{1}$, are positions of immediate offspring of a particle located at 0 .

Proposition 2.1: Assume that $\exists \kappa_{0}>0$ such that $m\left(\kappa_{0}\right)=1$. Then

$$
\psi(u) \leq \exp \left(-\kappa_{0} u\right) .
$$

Proof: Consider the following well-known martingale (see [4])

$$
W_{n}(\kappa)=\sum_{x \in G_{n}} \frac{\left.\exp \left[\kappa\left(Z_{x}-u\right)\right)\right]}{m(\kappa)^{n}} .
$$

Observe that $W_{0}(\kappa)=e^{-\kappa u}$. Let us stop $W_{n}\left(\kappa_{0}\right)$ at the moment of potential ruin $\theta(u)$. Clearly, $W_{\theta(u)}\left(\kappa_{0}\right) \geq 1$ and the martingale stopping theorem implies

$$
e^{-\kappa_{0} u}=W_{0}\left(\kappa_{0}\right) \geq E\left[W_{\theta(u)}\left(\kappa_{0}\right) \mid \theta(u)<\infty\right] P(\theta(u)<\infty) \geq \psi(u) . \bullet
$$

Next we prove a similar estimation for a model with Poisson stream of claims on a binary tree $\Gamma$ defined by the following conditions:

(a) for any fixed path $\mathbf{L} \in \mathcal{L}$ on the tree the points form a stationary Poisson flow of intensity $\lambda$;

(b) for any finite non-intersecting subgraphs $\Gamma_{1}, \Gamma_{2} \in \Gamma$ the numbers of points insides these subgraphs $N\left(\Gamma_{1}\right)$ and $N\left(\Gamma_{2}\right)$ are independent.

Let $\mathcal{I}(t)$ be the set of points of the graph that exist at the moment $t$ (i.e. within distance $t$ from the origin) and $I(t)=|\mathcal{I}(t)|$. Define

$$
L=\sup _{t>0}\left[\max _{i \leq I(t)} Z_{i}(t)\right], Z_{i}(t)=S_{i}(t)-c t
$$

where $S_{i}(t)=\sum_{t_{j} \in \mathbf{L}_{i}(t)} X_{t_{j}}, i \leq I(t)$ where $\mathbf{L}_{i}(t)$ is the path on the graph $\Gamma$ from the origin to the point $i \in \mathcal{I}(t)$.

It is convenient to write the ruin probability $\psi(0)$ as $\frac{1+\nu}{1+\rho}, 0 \leq \nu \leq \rho$. Observe that $\nu=0$ for one-dimensional time model.

Proposition 2.2: Assume that $\psi(0)=\frac{1+\nu}{1+\rho}<1$ (i.e., $0 \leq \nu<\rho$ ). Then the distribution function $\mathcal{F}_{L}(x)$ of maximal aggregated loss $L$ is majorized by the distribution function $\mathcal{F}_{\tilde{L}}(x)$ where $\tilde{L}$ is a random variable with the m.g.f.

$$
M_{\tilde{L}}(s)=\frac{\rho-\nu}{1+\rho-(1+\nu) M_{Y}(s)} .
$$

Here $M_{Y}(s)=\frac{M_{X}(s)-1}{\mu_{1} s}$. 
Proof: We use a representation of $L$ as a sum of random number $K$ of independent new record hights $L=Y_{1}+\ldots+Y_{K}$ :

$$
\mathbf{E} e^{s L}=\sum_{k=0}^{\infty} \sum_{\mathbf{L} \in \mathcal{L}} \int \ldots \int e^{s\left(y_{1}+\ldots+y_{k}\right)} P\left(\mathbf{L}, k, d y_{1}, \ldots, d y_{k}\right) .
$$

Here $P\left(\mathbf{L}, k, d y_{1}, \ldots, d y_{k}\right)$ is the joint distribution of the path $\mathbf{L} \in \mathcal{L}$ where the supremum in (2.4) is achieved, the number $k=k(\mathbf{L})$ of the record hights along the path $\mathbf{L}$, and the values of record hights $y_{1}, \ldots, y_{k}$. Observe that the for fixed $\mathbf{L}$ and $k(\mathbf{L}) \geq k$, random variables $Y_{j}, j \leq k$, are i.i.d. r.v. with distribution $P_{Y}(d y)$ and the m.g.f. $M_{Y}(s)=\frac{M_{X}(s)-1}{\mu_{1} s}$. Moreover, the distribution of $K$, i.e. the number of new record hights, is majorized by a geometrical distribution with parameter $\psi(0)=\frac{1+\nu}{1+\rho}$. It means that if we drop the condition that the path $\mathbf{L}$ is optimal and take the summation over all $\mathbf{L} \in \mathcal{L}$ then

$$
\sum_{\mathbf{L} \in \mathcal{L}} P(k(\mathbf{L}) \geq k) \leq \psi(0)^{k}
$$

Hence,

$$
\sum_{\mathbf{L} \in \mathcal{L}} P\left(k(\mathbf{L}) \geq k, d y_{1}, \ldots, d y_{k}\right) \leq \psi(0)^{k} \prod_{j=1}^{k} P_{Y}\left(d y_{j}\right) .
$$

This implies that

$$
\begin{gathered}
\mathbf{E} e^{s L} \leq 1+\sum_{k=1}^{\infty} \psi(0)^{k}\left(M_{Y}(s)^{k+1}-M_{Y}(s)^{k}\right)= \\
=\sum_{k=0}^{\infty} M_{Y}(s)^{k}\left(\psi(0)^{k}-\psi(0)^{k+1}\right)=(1-\psi(0)) \sum_{k=0}^{\infty} \psi(0)^{k} M_{Y}(s)^{k}=M_{\tilde{L}}(s) .
\end{gathered}
$$

A similar computation can be done for $\mathbf{E} f(L)$ with any increasing function of $L$, i.e. the distribution of the maximal aggregated loss $L$ is majorized by the distribution $\tilde{L}$ with the m.g.f. (2.5). This completes the proof.

A natural conjecture is that under broad assumptions, after rescaling $L=\bar{L} \sqrt{\gamma}$, $\rho=\frac{\bar{\rho}}{\sqrt{\gamma}}$, the parameter $\nu$ in representation $\psi(0)=\frac{1+\nu}{1+\rho}$, is rescaled asymptotically as

$$
\nu \approx \frac{\bar{\nu}}{\sqrt{\gamma}}, \gamma \rightarrow \infty
$$

At the moment we cannot offer a proof of (2.6). However, if true, this conjecture leads to the following result:

$$
\psi(u) \leq \exp \left(-\frac{2(\bar{\rho}-\bar{\nu}) \mu_{1} u}{(1+\bar{\nu}) m_{2}}\right) .
$$

To perform the diffusion approximation, we have to modify the model under consideration. Namely, we will assume a discrete time binary Markov model where the choice of the next policy depends on the current policy only. Denote by $p_{l m}(l, m=0,1)$ the probability to select the policy $\boldsymbol{\Pi}_{m}$ after $\boldsymbol{\Pi}_{l}$ and consider the case of a 'conservative investor' who sticks to a particular policy and decides to change it rarely. Formally, $\left(1-p_{l l}\right) \gamma \rightarrow \varpi>0 \quad(l=0,1)$ as $\gamma \rightarrow \infty$. Using the same scaling of the safety load 
$\rho=\frac{\bar{\rho}}{\sqrt{\gamma}}$ and the initial reserve $u=\mu_{1} \sqrt{\gamma}$ as $\gamma \rightarrow \infty$, one obtains a representation of potential ruin probability in terms of the branching diffusion [7]. We denote by $I(t)$ the number of particles in the branching diffusion with the constant drift $a$, variance $\sigma^{2}$ and intensity of branching $\varpi$ at the moment $t$, and by $Z_{i}(t)$ the position of $i$-th particle. Then the probability of potential ruin tends to the limit (cf. [7])

$$
\psi_{D}=\mathbf{P}\left(\sup _{t>0}\left[\max _{i \leq I(t)} Z_{i}(t)\right]>\frac{\mu_{1}}{\sqrt{\lambda m_{2}}}\right) .
$$

In contrast to one-dimensional case, one needs additional restrictions to exclude $\psi_{D} \equiv 1$.

\section{Multiple-Choice Potential Ruin Probabilities: A Dynamical System Approach}

In what follows we study the ruin problem on a tree in a diffusion approximation. As in Section 2, the branching diffusion process lives on $\mathbf{R}^{1}$, starts at the origin and has drift $a$, diffusion coefficient $\sigma$ and fission rate $\varpi$.

A natural parameter here is a number of generations (i.e., the number of fissions of the branching diffusion). In other words, we follow a given branch until the (random) time of $n$-th fission. As before, this model arises when we consider the case of conservative investors who do not consider changing a policy for a considerable period, but after an exponential time may think of a possible alternative.

The random distance between the position of a parent and the position of offspring at the moment of death $\xi$ can be treated as the overall balance between the two successive changes of the policy in the diffusion limit. It is easy to check that in the case of branching diffusion with drift $a$, diffusion $\sigma^{2}$ and the fission rate $\varpi, \xi$ is distributed as the difference

$$
\tau^{\prime}-\tau^{\prime \prime}
$$

of two independent exponential random variables $\tau^{\prime}$ with parameter $\lambda=\frac{a}{\sigma^{2}}+\left(\frac{a^{2}}{\sigma^{4}}+\right.$ $\left.2 \frac{\varpi}{\sigma^{2}}\right)^{1 / 2}$ and $\tau^{\prime \prime}$ with parameter $\mu=-\frac{a}{\sigma^{2}}+\left(\frac{a^{2}}{\sigma^{4}}+2 \frac{\varpi}{\sigma^{2}}\right)^{1 / 2}$ (cf. [10]).

In this context, it is natural to ask the following question. Suppose that investor's initial capital is $u>0$. How many paths of the diffusion tree will lead to the ruin of the investor (i.e., reach the level $u$ ) by the time of $n$-th fission (the total number of paths is again $\left.2^{n}\right)$ ? What is its limiting behaviour when $n \rightarrow \infty$ ? In this paper we provide some answers to these and other related questions although the situation needs further investigation. Our first remark is that if a 'ruinous' strategy exists $\forall u$ with the limiting probability one then, $\forall u>0$ and $k>1$, the limiting probability that there exist at least $k$ such strategies is also one.

We now introduce a formal setup for this result. Let $\mathfrak{F}$ and $\mathfrak{F}_{+}$be the set of the probability distributions on $\mathbf{R}^{1}$ and $\mathbf{R}_{+}^{1}=[0, \infty)$, respectively. We also use the notations $\mathfrak{F}_{+}^{l}=\mathfrak{F}_{+} \times \ldots \times \mathfrak{F}_{+}, \overline{\mathfrak{F}}_{+}^{k}=\left(\mathfrak{F}_{+}, \mathfrak{F}_{+}^{2}, \ldots, \mathfrak{F}_{+}^{k}\right)$.

Let us write down equations for the pair $\left(F_{n}, G_{n}\right) \in \mathfrak{F}_{+}^{2}$ of the distribution functions where

$$
\bar{F}_{n}(x)=1-F_{n}(x)=\mathbb{P}\left(\max Z_{\mathbf{L}} \geq x: \mathbf{L} \in \mathcal{L}_{n}\right),
$$

and $G_{n}=1-\bar{G}_{n}$, with

$$
\bar{G}_{n}(x)=\mathbb{P}\left(Z_{\mathbf{L}^{\prime}}, Z_{\mathbf{L}^{\prime \prime}} \geq x \text { for at least two different paths } \mathbf{L}^{\prime}, \mathbf{L}^{\prime \prime} \in \mathcal{L}_{n}\right) .
$$


Then

$$
F_{n+1}(x)=\left[\Lambda^{(1)} F_{n}\right](x), G_{n+1}(x)=\left[\Lambda^{(2)}\left(F_{n}, G_{n}\right)\right](x) .
$$

Here $\Lambda^{(1)}$ and $\Lambda^{(2)}$ are (non-linear) operators, $\Lambda^{(1)}: \mathfrak{F}_{+} \rightarrow \mathfrak{F}_{+}$and $\Lambda^{(2)}: \mathfrak{F}_{+}^{2} \rightarrow \mathfrak{F}_{+}$, given by

$$
\left[\Lambda^{(1)} F\right](x)=\int_{-\infty}^{x} d y p_{\xi}(x-y) F_{+}(y)^{2}
$$

and

$$
\begin{gathered}
{\left[\Lambda^{(2)}(F, G)\right](x)=\left[\Lambda^{(1)} F\right](x)} \\
+2 \int_{-\infty}^{x} d y p_{\xi}(x-y) F_{+}(y)\left(G_{+}(y)-F_{+}(y)\right) .
\end{gathered}
$$

Here $F_{+}=F \Theta$ where $\Theta$ is the indicator function of the non-negative half-line $[0, \infty)$.

The functional equations (3.5) and (3.6) are well-defined irrespectively of the specific form of the distribution of $\xi$.

Furthermore, $\forall k \geq 1$, define the $k$-tuple $\mathbf{F}_{n}=\left(F_{n}^{(1)}, \ldots, F_{n}^{(k)}\right)$ where $F_{n}^{(k)}(x)=$ $1-\bar{F}_{n}^{(k)}(x)$ and

$$
\bar{F}_{n}^{(k)}(x)=\mathbb{P}\left(Z_{\mathbf{L}^{j}} \geq x \text { for at least } k \text { different paths } \mathbf{L}^{1}, \ldots, \mathbf{L}^{k} \in \mathcal{L}_{n}\right) .
$$

Then vector-functions $\mathbf{F}_{n}$ and operators $\Lambda^{(l)}: \mathfrak{F}_{+}^{l} \rightarrow \mathfrak{F}_{+}$can be defined recursively:

$$
\begin{gathered}
\mathbf{F}_{n+1}=\Lambda \mathbf{F}_{n}=\left(\Lambda^{(1)} F_{n}^{(1)}, \ldots, \Lambda^{(k)}\left(F_{n}^{(1)}, \ldots, F_{n}^{(k)}\right)\right) ; \\
{\left[\Lambda^{(l)}\left(F^{(1)}, \ldots, F^{(l)}\right)\right](x)=\left[\Lambda^{(l-1)}\left(F^{(1)}, \ldots, F^{(l-1)}\right)\right](x)} \\
+\quad \sum_{\substack{0 \leq l_{1}, l_{2}: \\
l_{1}+l_{2}=l-1}} \int_{-\infty}^{x} d y p_{\xi}(x-y)\left(\left(F_{n-1}^{\left(l_{1}+1\right)}\right)_{+}-\left(F_{n-1}^{\left(l_{1}\right)}\right)_{+}\right) \\
\quad \times\left(\left(F_{n-1}^{\left(l_{2}+1\right)}\right)_{+}-\left(F_{n-1}^{\left(l_{2}\right)}\right)_{+}\right), l=1, \ldots, k,
\end{gathered}
$$

with $F_{n-1}^{(0)}(x) \equiv 0$.

We proof the following

Lemma 3.1: Let $F_{0}^{(1)}=\ldots=F_{0}^{(k)}=\Theta$. Then the $k$-tuple $\mathbf{F}_{n}=\left(F_{n}^{(1)}, \ldots, F_{n}^{(k)}\right)$ tends as $n \rightarrow \infty$ to a limit which is a fixed point of map $\Lambda=\left(\Lambda^{(1)}, \ldots, \Lambda^{(k)}\right)$, i.e. to a $k$-tuple $\mathbf{F}=\left(F^{(1)}, \ldots, F^{(k)}\right) \in \overline{\mathfrak{F}}_{+}^{k}$ with

$$
\Lambda \mathbf{F}=\mathbf{F} .
$$

Proof: In view of monotonicity of operator $\Lambda^{(1)}: 0 \leq \Lambda^{(1)} \Theta \leq \Theta$. This immediately implies the existence of a limit in the pointwise sense. The same monotonicity property holds for the operator $\Lambda^{(1)}+\ldots+\Lambda^{(k)}$. Finally, the limits are fixed points of respective operators.

Remark: Observe that the sets of all solutions of (3.10) is rather rich. Under the stability assumption

$$
\inf _{a>0} \mathbf{E} e^{a \xi} \leq \frac{1}{2}
$$


the set of all solutions of (3.5) was studied in ([9]). It is proved that there exists a linearly ordered continuum of solutions distinguished in terms of the asymptotics of the 'tails' (cf., again, [9]). Clearly, different solutions of (3.5) generate different solutions of the system (3.10).

Now we use the specific form of distribution of the random variable $\xi$ to simplify the functional equations (3.10) as follows. In this case the stability condition (3.11) takes the following form

$$
a<0, \quad \frac{a^{2}}{\sigma^{2}}>2 \varpi
$$

Lemma 3.2: If $\xi$ has the distribution (3.1) then any $k$-tuple of distribution functions $\mathbf{F}=\left(F^{(1)}, \ldots, F^{(k)}\right)$ satisfying $(3.10)$ solves the following recursive Cauchy problem:

$$
\begin{aligned}
& {\left[\mathbf{D} F^{(l+1)}\right](x)=\left[\mathbf{D} F^{(l)}\right](x)} \\
& -2 \frac{\varpi}{\sigma^{2}} \sum_{\substack{0 \leq l_{1}, l_{2}: \\
l_{1}+l_{2}=l-1}}\left(\left(F^{\left(l_{1}+1\right)}\right)_{+}(x)-\left(F^{\left(l_{1}\right)}\right)_{+}(x)\right) \\
& \quad \times\left(\left(F^{\left(l_{2}+1\right)}\right)_{+}(x)-\left(F^{\left(l_{2}\right)}\right)_{+}(x)\right), l=1, \ldots, k .
\end{aligned}
$$

with the initial conditions

$$
\left.F^{(l)}(x)\right|_{x=0}=0, \quad l=1, \ldots, k .
$$

Here, $\mathbf{D}$ is the second order differential operator

$$
\mathbf{D}=\frac{d^{2}}{d x^{2}}-\frac{2 a}{\sigma^{2}} \frac{d}{d x}-\frac{2 \varpi}{\sigma^{2}} .
$$

A slightly more convenient form of Lemma 3.2 is

Lemma 3.3: $\operatorname{Set}^{(0)}(x) \equiv 0$ and

$$
u^{(l)}(x)=F^{(l)}(x)-F^{(l-1)}(x), v^{(l)}(x)=\frac{d}{d x} u^{(l)}(x), x \geq 0 .
$$

Then $\left(u^{(l)}(x), v^{(l)}(x)\right), \quad l=1, \ldots, k$, obey

$$
\begin{gathered}
\frac{d}{d x} u^{(l)}(x)=v^{(l)}(x), x>0, \\
\frac{d}{d x} v^{(l)}(x)=2 \frac{a}{\sigma^{2}} v^{(l)}(x)-2 \frac{\varpi}{\sigma^{2}} u^{(l)}(x) \\
\left.-2 \frac{\varpi}{\sigma^{2}} \quad \sum_{0 \leq l_{1}, l_{2}:}^{\left(l_{1}+1\right)}\right)_{+}(x)\left(u^{\left(l_{2}+1\right)}\right)_{+}(x), x>0, \\
l_{1}+l_{2}=l-1
\end{gathered}
$$

with the initial conditions

$$
u^{(l)}(0)=0, l=1, \ldots, k .
$$

Moreover, functions $u^{(l)}$ remain non-negative: $u^{(l)}(x) \geq 0, x \geq 0$. 
The proof of Lemmas 3.2, 3.3 is straightforward and based on explicit form of kernel $p_{\xi}(x-y)$ (cf. [9]).

We conclude that the solution to invariance equations (3.8) are non-unique. The origin of this non-uniqueness is the long-time memory (lack of renovation) of the stochastic dynamics.

In the case $k=2$, a number of aspects of the theory is simplified. If we set

$$
\begin{gathered}
u^{(1)}(x)=F(x), v^{(1)}(x)=\frac{d}{d x} u^{(1)}(x), x \geq 0, \\
u^{(2)}(x)=G(x)-F(x), v^{(2)}(x)=\frac{d}{d x} u^{(2)}(x), x \geq 0,
\end{gathered}
$$

then $\left(u^{(l)}(x), v^{(l)}(x)\right), l=1,2$, obey

$$
\begin{gathered}
\frac{d}{d x} u^{(l)}(x)=v^{(l)}(x), x>0, \\
\frac{d}{d x} v^{(1)}(x)=2 \frac{a}{\sigma^{2}} v^{(1)}(x)+2 \frac{\varpi}{\sigma^{2}}\left(u^{(1)}(x)-u^{(1)}(x)^{2}\right), x>0, \\
\frac{d}{d x} v^{(2)}(x)=2 \frac{a}{\sigma^{2}} v^{(2)}(x) \\
-2 \frac{\varpi}{\sigma^{2}}\left(u^{(2)}(x)-2 u^{(l)}(x) u^{(2)}(x)\right), x>0,
\end{gathered}
$$

with the initial conditions

$$
u^{(l)}(0)=0, l=1,2 .
$$

\section{A Phase Portrait Analysis}

We now proceed with an analysis of the phase portrait of the dynamical system (3.14)(3.15). There are two equilibrium points of this system: the origin $\mathbf{0} \in \mathbb{R}^{2 k}$ and $\mathbf{J}=$ $(1,0 \ldots, 0)$. The first point is a saddle, while the type of the second point depends on values $a, \sigma$ and $\varpi$. $\mathbf{J}$ is an attracting node under the stability conditions (3.10)

and an (attracting) clockwise focus if this condition is violated. Therefore, a solution $\left(F^{(1)}, \ldots, F^{(k)}\right)$ to $\Lambda\left(F^{(1)}, \ldots, F^{(k)}\right)=\left(F^{(1)}, \ldots, F^{(k)}\right)$ such that $0 \leq F^{(l)}(x) \leq 1$ and $\lim _{x \rightarrow \infty} F^{(l)}(x)=1$ may exists only under condition (3.10). In fact, the linearisation of system (3.14)-(3.15) around $\mathbf{0}$ yields the matrix

$$
\mathbf{A}_{\mathbf{O}}=\left(\begin{array}{cccccccc}
0 & 1 & 0 & 0 & \ldots & \ldots & 0 & 0 \\
2 \frac{\varpi}{\sigma^{2}} & 2 \frac{a}{\sigma^{2}} & 0 & 0 & \ldots & \ldots & 0 & 0 \\
0 & 0 & 0 & 1 & \ldots & \ldots & 0 & 0 \\
0 & 0 & 2 \frac{\varpi}{\sigma^{2}} & 2 \frac{a}{\sigma^{2}} & \ldots & \ldots & 0 & 0 \\
\ldots & \ldots & \ldots & \ldots & \ldots & \ldots & \ldots & \ldots \\
0 & 0 & 0 & 0 & \ldots & \ldots & 0 & 1 \\
0 & 0 & 0 & 0 & \ldots & \ldots & 2 \frac{\varpi}{\sigma^{2}} & 2 \frac{a}{\sigma^{2}}
\end{array}\right)
$$


while around $\mathbf{J}$ the matrix

$$
\mathbf{A}_{\mathbf{J}}=\left(\begin{array}{cccccccc}
0 & 1 & 0 & 0 & \ldots & \ldots & 0 & 0 \\
-2 \frac{\varpi}{\sigma^{2}} & 2 \frac{a}{\sigma^{2}} & 0 & 0 & \ldots & \ldots & 0 & 0 \\
0 & 0 & 0 & 1 & \ldots & \ldots & 0 & 0 \\
0 & 0 & -2 \frac{\varpi}{\sigma^{2}} & 2 \frac{a}{\sigma^{2}} & \ldots & \ldots & 0 & 0 \\
\ldots & \ldots & \ldots & \ldots & \ldots & \ldots & \ldots & \ldots \\
0 & 0 & 0 & 0 & \ldots & \ldots & 0 & 1 \\
0 & 0 & 0 & 0 & \ldots & \ldots & -2 \frac{\varpi}{\sigma^{2}} & 2 \frac{a}{\sigma^{2}}
\end{array}\right)
$$

At $\mathbf{0}$, the $k$ directions generating a $k$-dimensional unstable manifold are along the vectors

$$
\left(1, \frac{a}{\sigma^{2}}+\left(\frac{a^{2}}{\sigma^{4}}+2 \frac{\varpi}{\sigma^{2}}\right)^{1 / 2}, 0, \ldots, 0\right), \ldots,\left(0,0,0, \ldots, 1, \frac{a}{\sigma^{2}}+\left(\frac{a^{2}}{\sigma^{4}}+2 \frac{\varpi}{\sigma^{2}}\right)^{1 / 2}\right)
$$

(these are simply eigen-vectors of $\mathbf{A}_{\mathbf{0}}$ with the positive eigen-value $\frac{a}{\sigma^{2}}+\left(\frac{a^{2}}{\sigma^{4}}+2 \frac{\varpi}{\sigma^{2}}\right)^{1 / 2}$ ), while the $k$ directions generating a $k$-dimensional stable manifold are along the vectors

$$
\left(-1,-\frac{a}{\sigma^{2}}+\left(\frac{a^{2}}{\sigma^{4}}+2 \frac{\varpi}{\sigma^{2}}\right)^{1 / 2}, 0, \ldots, 0\right), \ldots,\left(0,0,0, \ldots,-1,-\frac{a}{\sigma^{2}}+\left(\frac{a^{2}}{\sigma^{4}}+2 \frac{\varpi}{\sigma^{2}}\right)^{1 / 2}\right)
$$

(these are eigen-vectors of $\mathbf{A}_{\mathbf{0}}$ with the negative eigen-value $\left.\frac{a}{\sigma^{2}}-\left(\frac{a^{2}}{\sigma^{4}}+2 \frac{\varpi}{\sigma^{2}}\right)^{1 / 2}\right)$, At $\mathbf{J}$, under condition (3.10), there are $2 k$ stable directions; $k$ of them generate a $k$-dimensional stable manifold and are along the vectors

$$
\left.\left(1, \frac{a}{\sigma^{2}}-\left(\frac{a^{2}}{\sigma^{4}}-2 \frac{\varpi}{\sigma^{2}}\right)^{1 / 2}\right), 0, \ldots, 0\right), \ldots,\left(0,0,0, \ldots, 1, \frac{a}{\sigma^{2}}-\left(\frac{a^{2}}{\sigma^{4}}-2 \frac{\varpi}{\sigma^{2}}\right)^{1 / 2}\right)
$$

(these are eigen-vectors with the negative eigen-value $\left.\frac{a}{\sigma^{2}}-\left(\frac{a^{2}}{\sigma^{4}}-2 \frac{\varpi}{\sigma^{2}}\right)^{1 / 2}\right)$, whereas another $k$ directions generate a $k$-dimensional stable manifold and are along the vectors

$$
\left(1, \frac{a}{\sigma^{2}}+\left(\frac{a^{2}}{\sigma^{4}}-2 \frac{\varpi}{\sigma^{2}}\right)^{1 / 2}, 0, \ldots, 0\right), \ldots,\left(0,0,0, \ldots, 1, \frac{a}{\sigma^{2}}+\left(\frac{a^{2}}{\sigma^{4}}-2 \frac{\varpi}{\sigma^{2}}\right)^{1 / 2}\right)
$$

(these are the eigen-vectors with the negative eigen-value $\frac{a}{\sigma^{2}}+\left(\frac{a^{2}}{\sigma^{4}}-2 \frac{\varpi}{\sigma^{2}}\right)^{1 / 2}$ ).

An analysis of the phase portrait of the dynamical system (3.14)-(3.15) in $\mathbf{R}^{2 k}$ shows that under stability condition (3.10) there exists a $k$-parameter family of solutions confined to the domain

$$
\mathcal{D}^{k}=\left(u^{(j)} \geq 0, \sum_{l=1}^{j} u^{(l)} \leq 1, \sum_{l=1}^{j} v^{(l)} \geq 0, j=1, \ldots, k\right)
$$

and entering node $\mathbf{J}$ as $x \rightarrow \infty$. These solutions possess a natural ordering which is specified below. In addition, there exists an unique 'isolated' solution which can be treated as 'extreme', in the sense explained below.

Correspondingly, the original system (3.14)-(3.15) has a $k$-dimensional continuum of solutions $\underline{F}=\left(F^{(1)}, \ldots, F^{(k)}\right)$ and an isolated solution $\underline{F}_{0}=\left(F_{0}^{(1)}, \ldots, F_{0}^{(k)}\right)$. The isolated solution is maximal in the point-wise sense: $F_{0}^{(j)} \geq F^{(j)}\left(\right.$ and $\left.F_{0}^{(j)} \not \equiv F^{(j)}\right)$. 
A straightforward parameter labelling a trajectory of (3.14)-(3.15) is an initial data vector $\underline{v}(0)=\left(v^{(1)}(0), \ldots, v^{(k)}(0)\right)$, with

$$
v^{(j)}(0) \geq 0, \quad j=1, \ldots, k .
$$

More precisely, the following fact holds true:

Theorem 4.1: Assume that the stability condition (3.10) is fulfilled. Then there exists a vector $\underline{r}=\left(r^{(1)}, \ldots, r^{(k)}\right)$ with $0<r^{(1)}<\ldots<r^{(k)}<1$ such that for any $\underline{v}(0)=\left(v^{(1)}(0), \ldots, v^{(k)}(0)\right)$ satisfying the conditions

$$
0 \leq \sum_{l=1}^{j} v^{(l)}(0) \leq r^{(l)}, l=1, \ldots, k
$$

there exists a unique solution $\underline{u}(x)=\left(u^{(1)}(x), \ldots, u^{(k)}(x)\right), \underline{v}(x)=$

$\left(v^{(1)}(x), \ldots, v^{(k)}(x)\right), x>0$, of problem (3.14)-(3.15) confined to the above domain $\mathcal{D}^{k}$ and entering $\mathbf{J}$.

A more natural label for the trajectory is related to the detailed description of the behaviour of the trajectory near the point $u^{(1)}=\ldots=u^{(k)}=v^{(1)}=\ldots=v^{(k)}=0$. It will be introduced below in Theorem 4.2 in the particular case $k=2$.

For $k=2$ the system (3.17) has two equilibrium points in $\mathbf{R}^{4}$ : a saddle point $\mathbf{0}=(0,0,0,0)$ and $\mathbf{J}=(1,0,0,0)$ (an attracting node under conditions (3.10). Furthermore, the equations for $\left(u^{(1)}, v^{(1)}\right)$ are autonomous and generate a dynamical system on a plane. The asymptotic behaviour of $F(x)$ as $x \rightarrow \infty$ was analysed in [9],[10]. In particular, under condition (3.10) $0<F(x)<1, \frac{d}{d x} F(x)>0$ for all $x>0$ and $\lim _{x \rightarrow \infty} F(x)=1$. Given $F(x)$ (i.e., a trajectory $\left.\left(u^{(1)}(x), v^{(1)}(x)\right), x>0\right)$ the equations for $G(x)-F(x)$ (i.e., $\left(u^{(2)}(x), v^{(2)}(x)\right), x>0$ ) are linear, albeit with the coefficient $u^{(1)}(x)$, depending on 'time' $x$. An elementary bound $u^{(1)}(x)+u^{(2)}(x)<1$ implies that the last summand in RHS of (3.17) obeys the bounds

$$
-u^{(2)}(x)+2\left(u^{(2)}(x)\right)^{2}<u^{(2)}(x)-2 u^{(1)}(x) u^{(2)}(x)<u^{(2)}(x), x>0 .
$$

Consider vector fields $\left(U^{(l)}(u, v), V^{(l)}(u, v)\right), l=2,3,4$ on the strip $\mathbf{S}=\{0 \leq u \leq 1, v \geq$ $0\}$ in the $u, v$-plane given by

$$
U^{(l)}(u, v)=v, l=2,3,4
$$

and

$$
\begin{gathered}
V^{(3)}(u, v)=2 \frac{a}{\sigma^{2}} v+2 \frac{\varpi}{\sigma^{2}} u, \\
V^{(4)}(u, v)=2 \frac{a}{\sigma^{2}} v+2 \frac{\varpi}{\sigma^{2}}\left(-u+2 u^{2}\right), \\
V^{(2)}(u, v, x)=2 \frac{a}{\sigma^{2}} v+2 \frac{\varpi}{\sigma^{2}}\left(u-2 u^{(1)}(x) u\right) .
\end{gathered}
$$

They determine, respectively, a linear system

$$
\frac{d}{d x} u^{(3)}(x)=v^{(3)}(x), \frac{d}{d x} v^{(3)}(x)=2 \frac{a}{\sigma^{2}} v^{(3)}(x)+2 \frac{\varpi}{\sigma^{2}} u^{(3)}(x),
$$

a non-linear (albeit autonomous) system

$$
\frac{d}{d x} u^{(4)}(x)=v^{(4)}(x), \frac{d}{d x} v^{(4)}(x)=2 \frac{a}{\sigma^{2}} v^{(4)}(x)+2 \frac{\varpi}{\sigma^{2}}\left(-u^{(4)}(x)+2\left(u^{(4)}(x)\right)^{2}\right),
$$


and linear (albeit non-autonomous) system

$$
\frac{d}{d x} u^{(2)}(x)=v^{(2)}(x), \frac{d}{d x} u^{(2)}(x)=2 \frac{a}{\sigma^{2}} v^{(2)}(x)+2 \frac{\varpi}{\sigma^{2}}\left(u^{(2)}(x)-u^{(1)}(x) u^{(2)}(x) .\right.
$$

According to $(4.5), U^{(3)}(u, v)=U^{(4)}(u, v)=U^{(2)}(u, v)$, and

$$
V^{(4)}(u, v) \leq V^{(2)}(u, v) \leq V^{(3)}(u, v) .
$$

This means that the trajectories of system (4.8) lie above and those of (4.9) below the trajectories of system (4.10) (provided of course, that all three trajectories are issued from the same initial point $(u(0), v(0)) \in \mathbf{S})$. Clearly, the system (4.8) has a saddle point at $\mathbf{J}$. It is easy to obtain the eigenvalues $\lambda_{1}=\frac{a}{\sigma^{2}}+\left(\frac{a^{2}}{\sigma^{4}}+2 \frac{\varpi}{\sigma^{2}}\right)^{1 / 2}$ and $\lambda_{2}=$ $\frac{a}{\sigma^{2}}-\left(\frac{a^{2}}{\sigma^{4}}+2 \frac{\varpi}{\sigma^{2}}\right)^{1 / 2}$ with eigenvectors $\left(1, \lambda_{1}\right)$ and $\left(1, \lambda_{2}\right)$, respectively. The first direction determines the attracting stable nodal separatrix, and there is a continuum of solutions of (4.8) which enters node $\mathbf{J}$ along this vector. The second direction determines a repelling stable nodal separatrix. It is intuitively clear that starting in a neighbourhood of the origin $\mathbf{0}$, the trajectories of (4.10) behave at first like the trajectories of the linear system (4.8), i.e. go from the vertical axis $u \equiv 0$ to the unstable direction $\left(1, \frac{a}{\sigma^{2}}+\left(\frac{a^{2}}{\sigma^{4}}+2 \frac{\varpi}{\sigma^{2}}\right)^{1 / 2}\right)$. On the other hand, $u^{(1)}(x) \approx 1$ for 'big times' $x$, in this case under condition (3.10), $\mathbf{J}$ becomes an attractive node and trajectories enters $\mathbf{J}$ along the attracting direction $\left(1, \lambda_{1}\right)$. It means that $v^{(2)}(x)$ becomes negative as $x \rightarrow \infty$. However, the sum $v^{(1)}(x)+v^{(2)}(x) \geq 0$. If the condition (3.10) is violated, $\mathbf{J}$ becomes a stable (attracting) clockwise focus. It is easy to check that a solution satisfying conditions $u^{(2)}(x)>0, v^{(1)}(x)+v^{(2)}(x) \geq 0$ for all $x>0$ cannot exist.

We make precise these heuristic arguments by comparing the trajectories of dynamical systems (4.8)-(4.10) as in the book [1]. Thus, we have

Theorem 4.2: For $k=2$, assume that stability condition (3.10) is fulfilled. Then there exist constants $r^{(1)}$ and $r^{(2)}, 0<r^{(1)} \leq r^{(2)}<1$, such that for any initial point $u^{(1)}(0)=u^{(2)}(0)=0$ and $\left(v^{(1)}(0), v^{(2)}(0)\right)$ with

$$
0<v^{(1)}(0) \leq r^{(1)}, 0<v^{(1)}(0)+v^{(2)}(0) \leq r^{(2)}
$$

there exists a solution $\left(u^{(1)}(x), v^{(1)}(x), u^{(2)}(x), v^{(2)}(x)\right), x>0$, to problem (3.17) such that the corresponding trajectory is confined to the strip $\mathcal{D}^{2}=\left\{u^{(1)}, v^{(1)}, u^{(2)}, v^{(2)}\right.$ : $\left.u^{(1)}, u^{(2)} \geq 0, v^{(1)} \geq 0, v^{(1)}+v^{(2)} \geq 0\right\}$ and enters the node $\mathbf{J}$. These solutions, labelled by pairs $\left(v^{(1)}(0), v^{(2)}(0)\right)$, form a two-dimensional continuum family that is partially ordered in accordance with the partial order of pairs $\left(v^{(1)}(0), v^{(2)}(0)\right)$. For each initial data with $0<v^{(1)}(0)<r^{(1)}, 0<v^{(1)}(0)+v^{(2)}(0)<r^{(2)}$, the solution obeys

$$
\lim _{x \rightarrow \infty} \frac{v^{(l)}(x)}{1-u^{(l)}(x)}=\alpha, l=1,2,
$$

whereas if $v^{(1)}(0)=r^{(1)}, v^{(1)}(0)+v^{(2)}(0)=r^{(2)}$, then

$$
\lim _{x \rightarrow \infty} \frac{v^{(l)}(x)}{1-u^{(l)}(x)}=\beta, l=1,2 .
$$

Furthermore, the solution with asymptotics (4.11) obeys

$$
\lim _{x \rightarrow \infty}\left(\frac{v^{(l)}(x)}{1-u^{(l)}(x)}-\alpha-c_{l} \exp [(\beta-\alpha) x]\right)=0, l=1,2 .
$$


Here $\underline{c}=\left(c_{1}, c_{2}\right)$ is a real vector providing an alternative labelling of solutions (i.e., the correspondence $\left(v^{(l)}(0), v^{(2)}(0)\right) \leftrightarrow \underline{c}$ is one-to-one which preserves partial ordering).

On the other hand, if the initial data $\left(u^{(1)}(0), v^{(1)}(0), u^{(2)}(0), v^{(2)}(0)\right)$ are such that $u^{(1)}(0)=u^{(2)}(0)=0$ but $v^{(1)}(0)>r^{(1)}$ or $v^{(1)}(0)+v^{(2)}(0)>r^{(2)}$ then there is no solution such that the corresponding trajectory is confined to the domain $\mathcal{D}^{2}$.

The 'extreme' case where $u^{(1)}(0)=u^{(2)}(0)=0, v^{(2)}(0)=r^{1}$ and $v^{(2)}(0)=r^{2}-r^{1}$ is discussed in

Theorem 4.3: For $k=2$, assume the condition (3.10) and suppose that $u^{(1)}(0)=$ $u^{(2)}(0)=0, v^{(1)}(0)=r^{1}$ and $v^{(2)}(0)=r^{2}-r^{1}$, where constants $r^{2}, r^{1}$ are introduced in Theorem 4.2. Then there exists a solution $\left(u_{0}^{(1)}(x), v_{0}^{(1)}(x), u_{0}^{(2)}(x), v_{0}^{(2)}(x)\right), x>0$, to (3.17) which is confined into $\mathcal{D}^{2}$. This solution is maximal in the following sense: $\forall$ solution $\left(u^{(1)}(x), v^{(1)}(x), u^{(2)}(x), v^{(2)}(x)\right)$ from Theorem 4.2,

$$
u_{0}^{(1)}(x) \geq u^{(1)}(x), u_{0}^{(1)}(x)+u_{0}^{(2)}(x) \geq u^{(1)}(x)+u^{(2)}(x), x>0 .
$$

Finally, for any value of $k$ the following statement holds:

Theorem 4.4: If the inequality sign in (3.10) is reversed then there is no solution to (3.17) which is confined into $\mathcal{D}^{2}$.

The proof of Theorems 4.1-4.4 follows from inspection of the above phase portrait.

Acknowledgements. Y. Suhov thanks IHES, Bures-sur-Yvette, for the hospitality during visits in 2002-2003.

\section{References}

[1] Andronov A.A., Leontovich E.A., Gordon I.I. and Maier A.G., Theory of Bifurcations of Dynamical Systems on a Plane, Jerusalem, Israel Program of Scientific Translation 1971

[2] Asmussen S., Applied Probability and Queues, John Wiley \& Sons, New York 1987

[3] Asmussen S., Ruin Probabilities, World Scientific, Singapore 2000

[4] Biggins J., Lindley-type equations in the branching random walk, Stoch. Proc. Appl. 75:1 (1998), 105-133.

[5] Borodin A.N., Salminen P., Handbook of Brownian motion. Facts and Formulae, Birkhäuser-Verlag, Berlin 1996

[6] Bowers N., Gerber H., Hickman D. and Nesbitt C., Risk Theory, Society of Actuaries, USA 1986

[7] Duffield N.G., Kelbert M. and Suhov Y., The branching diffusion approximation for a class of a synchronized queueing network, In: Statistics and Control of Stochastic Processes, Steklov Mathematical Institute (ed. by Y.Kabanov, B.Rozovsky and A.Shiryaev), World Scientific, Singapore (1997), 13-40.

[8] Kelbert M., Rachev S. and Suhov Y., Markov-splitted random fields on a tree, with applications. In: Analytical Methods in Applied Probability. In Memory of Fridrih Karpelevich, (ed. by Y.Suhov), AMS Translations, Ser.2 AMS, Providence 2002.

[9] Karpelevich F., Kelbert M. and Suhov Y., Higher-order Lindley equations, Stoch. Proc. Appl. 53 (1994), 65-96.

[10] Kelbert M. and Suhov Y., The Markov branching random walk and systems of reactiondiffusion (Kolmogorov-Petrovskii-Piskunov) equations, Commun. Math. Physics 167 (1995), 607-634. 


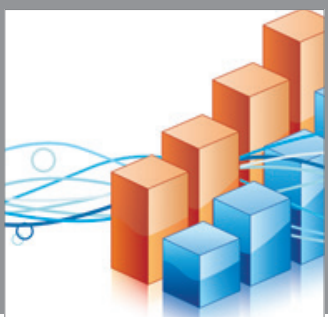

Advances in

Operations Research

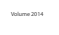

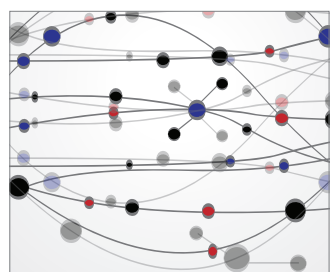

\section{The Scientific} World Journal
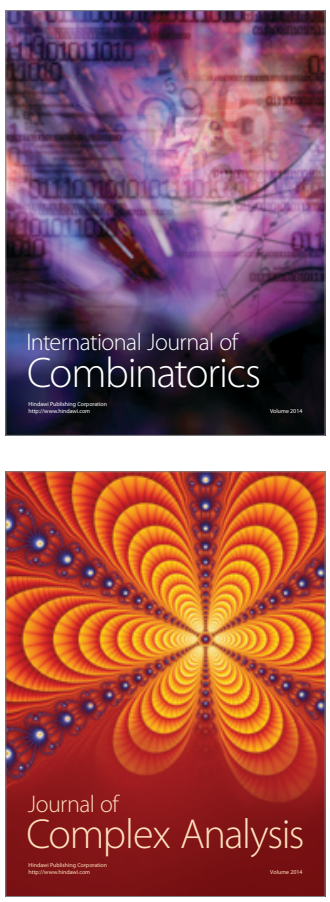

International Journal of

Mathematics and

Mathematical

Sciences
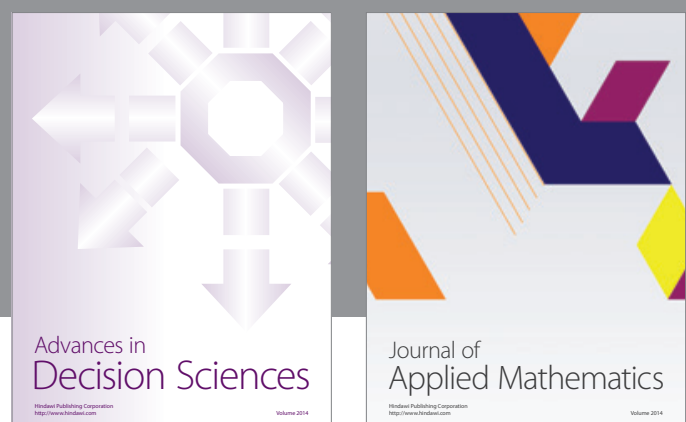

Journal of

Applied Mathematics
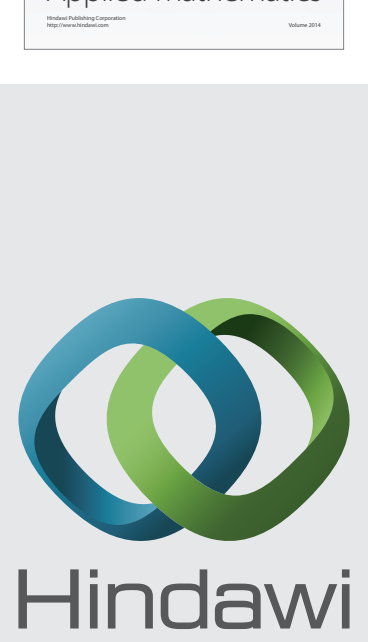

Submit your manuscripts at http://www.hindawi.com
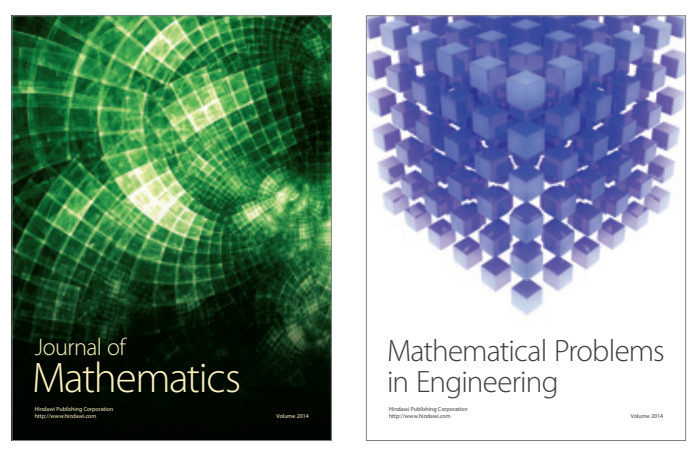

Mathematical Problems in Engineering
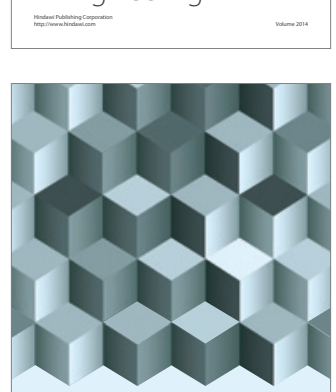

Journal of

Function Spaces
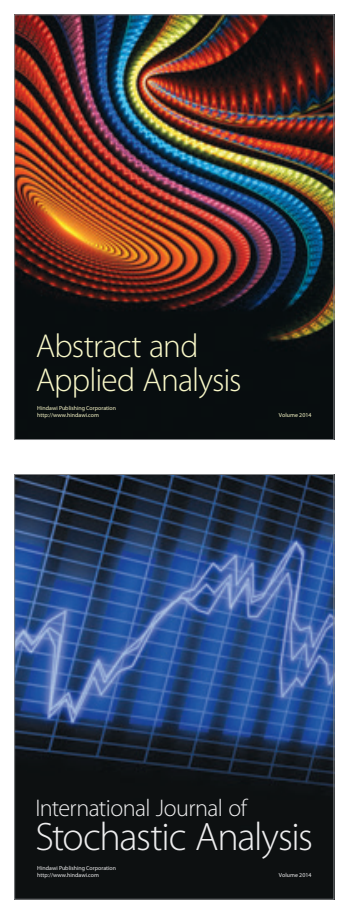

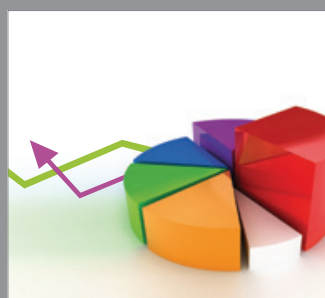

ournal of

Probability and Statistics

Promensencen
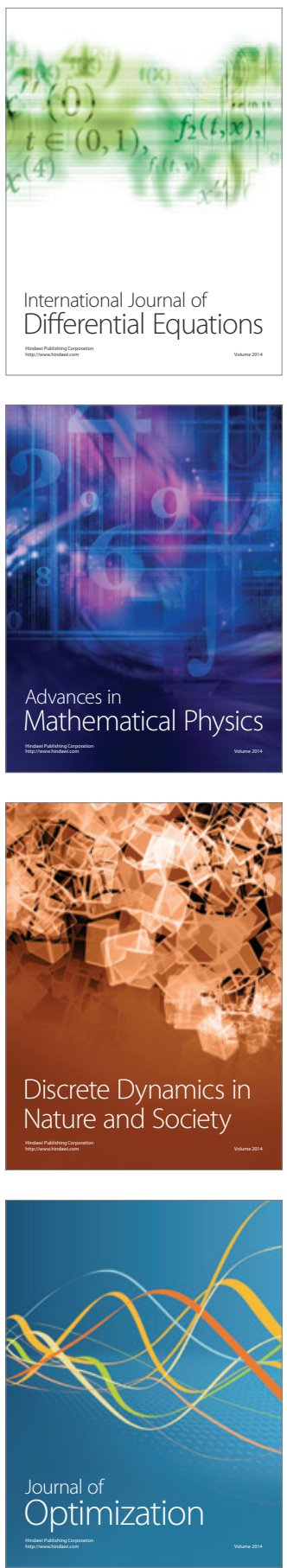